\title{
Development of a web-based land-use mapping
}

Romeo L. Jorda, Jr. ${ }^{1,2 *}$, Edmon O. Fernandez ${ }^{1,2}$, Jessica S. Velasco ${ }^{1,2}$, Maria Victoria C. Padilla ${ }^{1,2}$, Shiela Mae P. Agoylo ${ }^{1}$, Mary Joy A. Bultron ${ }^{1}$, Ailyn Joyce O. Clamor ${ }^{1}$, Jaishree Keith M. Monzaga $^{1}$, Catherine Rose R. Rosales ${ }^{1}$, Cleodelaine S. Salvador ${ }^{1}$ and Lean Karlo S. Tolentino ${ }^{1,2,3}$ Department of Electronics Engineering, Technological University of the Philippines, Manila, Philippines ${ }^{1}$ Center for Engineering Design, Fabrication, and Innovation, College of Engineering, Technological University of the Philippines, Manila, Philippines ${ }^{2}$

University Extension Services Office, Technological University of the Philippines, Manila, Philippines ${ }^{3}$

Received: 20-December-2020; Revised: 22-February-2021; Accepted: 24-February-2021

(C2021 Romeo L. Jorda, Jr et al. This is an open access article distributed under the Creative Commons Attribution (CC BY) License, which permits unrestricted use, distribution, and reproduction in any medium, provided the original work is properly cited.

\begin{abstract}
Local Government Units (LGUs) in the Philippines are mandated to conduct land classification mapping of their areas which brings up an issue about a time-consuming and expensive data gathering. This paper aims at achieving a land use classification system that correlates with the traditional way of mapping of LGUs. This study introduces the development of a web application that can classify land use (Agricultural, Commercial, Residential, and Industrial) in different areas of Greater Manila. The system involves the utilization of freely accessible satellite images from Google Maps and benchmark dataset from the EuroSAT dataset for the categorization of the areas through deep learning using Convolutional Neural Network (CNN). Evaluation results show that the system in a web application could help with the mapping of the Greater Manila area with the web application being simple yet informative and efficient.
\end{abstract}

\section{Keywords}

CNN, Land use classification, Satellite imagery, Web application.

\section{Introduction}

The Philippines has a policy about land classification and urban planning as stated in Republic Act No. 7279 Article 4, "Land Use, Inventory, Acquisition and Disposition" [1]. Land use for every city and municipal governments requires an inventory. Frequent monitoring can help local government units to have immediate action for problems such as an appropriate settlement allocation. As a result, every local government unit in the country should monitor the land use over their area for proper distribution of their cities' resources. Classification of land use in the areas of the Philippines is a challenge, especially because of the time-consuming and costly traditional and manual way of land mapping of government institutions. Also, studying land use in the country is a challenge with the limited data of ground truths and references that are publicly available because most datasets are confidentially owned by administrative bodies and cannot be accessed publicly.

*Author for correspondence 221
One solution to this limitation of information is with the use of satellite imagery. The rapid progresses in remote sensing technology, and the reduction of acquisition costs, makes a large bulk of images of the Earth readily available for academic and commercial purpose due to efforts made by governmental agencies such as National Aeronautics and Space Administration's (NASA) Landsat and European Space Agency's (ESA) Copernicus [2, 3]. With access to such data, applications in different domains, including urban development, can be realized [4].

With these means, the general objective of this study is to develop a web application for land use classification. This research focuses on processing the acquired publicly available satellite images through CNN deep learning method to fully utilize these openly accessible remote sensing data and integrating this system to a web application which can help LGUs and the public with the purpose of providing land classification for zoning reference and to help improve the land use classification problem of the country. 


\section{Related works}

To develop the land use classification system, previous policy of land use classification in the Philippines and studies about satellite imagery applications and land use and land cover classification is studied by the researchers working with different views. Furthermore, open source for satellite images, remotely sensed aerial and satellite image datasets, and classification methods for land use classification are presented [4].

Technology nowadays allows data from different sources to be studied to be able to help in solving world problems or even the smallest problem in the society. With multiple sources, data can be found everywhere [5]. One of these sources of data can be from satellites.

Satellite imaging was used before only for military activities, but today due to advanced technology, satellite images are also used for different applications. Satellite imaging with radar can be used nowadays for climate monitoring, coastal monitoring, land use, forestry and agriculture, natural resources exploitation, map updating and compiling, marine environment, natural disaster, etc. These shows that satellite imagery has a wide range of applications that have great impact on our daily lives [6].

In 2015, Gopinath et al. [7] aimed the monitoring of different levels of drought with the use of geospatial techniques. Satellite data are obtained from MODISTerra satellite of the NASA. Normalized Difference Vegetation Index (NDVI) is the major parameter used in measuring the vegetation health and for over 13 years of the Kerala state, the mean NDVI is calculated. Based on which the drought risk classification is done, over the same period, NDVI daily anomalies were determined from long term mean NDVI. Vulnerable to drought are areas with high negative NDVI anomaly. Levels of drought risk can be identified using Land Use/Land Cover data. The drought risk for different crops is given by covering daily NDVI Anomaly drought risk map on land use/land cover map.

With the launch of the first Philippine microsatellite - DIWATA-1, which has been built to undertake scientific earth observation missions related to weather observation, environmental monitoring, and disaster risk management [8], the proponents looked into one of the applications of satellite imagery which is the study of land use classification in certain areas in the Philippines. With this, the following are some of the open access portals that provide satellite imagery data:

\section{Copernicus open access hub}

An open access portal developed by ESA, which steps up efforts in improving Earth observation within its Copernicus program. Under this program, ESA operates a series of satellites known as Sentinels [4]. Data from these satellites are often used for land use classification for it obtains imagery over land and coastal waters. Spatial resolutions of these satellites range from $10 \mathrm{~m}$ to $60 \mathrm{~m}$.

\section{USGS earth explorer}

An online tool developed by the United States Geological Survey (USGS) which is the primary provider of Landsat Satellite image data [9]. Landsat is also often used in land use classification which has a spatial resolution that ranges from $30 \mathrm{~m}$ to $60 \mathrm{~m}$. USGS Earth Explorer also provides data from other satellites.

\section{Google maps}

A free-to-use, web-based mapping service that combines conventional cartographic maps with satellite imagery and high-resolution aerial photography [10]. Satellite images come from various satellites including Copernicus' Sentinel.

Along with the use of satellite data, deep learning methods can be utilized in the classification of the images. Deep learning permits computational models that consist of several processing layers to study depictions of data with several stages of abstraction which have enhanced diverse recognitions and detections such as speech and objects. Deep convolutional networks and recurrent networks have carried innovations in handing out images, video, speech, and audio for the former, and on sequential data such as text and speech for the latter [11].

CNN is deep neural networks that do image recognition or classification. $\mathrm{CNN}$ is improving every year and is used for different services. Nowadays, it is prominent for the programmer in the aspect of image processing because of the accuracy of an artificial intelligence of it to recognize everything [12]. Neural networks can be fast in producing results and can identify connections between seeing data instances that humans can hardly see [13]. In this study, classification of the satellite images that are obtained was implemented. 
In 2016, Jean et al. [14] exhibit how a convolutional neural system can be instructed to characterize picture attributes that can represent as meager as $75 \%$ of the change in money related outcomes at neighborhood level utilizing study data and satellite data from five African nations - Nigeria, Tanzania, Uganda, Malawi, and Rwanda. Their paper shows an exact way to deal with assessing utilization and the benefits of high-goal satellite imaging that would be financially savvy and adaptable. The methodology, which just needs transparently open data, can turn endeavors in rising countries to screen and target destitution. It likewise shows how solid AI strategies can be actualized in a situation with confined preparing data and proposes wide imminent applications in a few science fields.

Training a CNN from scratch could take a lot of time. For this reason, transfer learning/fine tuning an existing network would be more efficient. A study at the University of San Diego [15] have evaluated pretrained CNNs using basic transfer learning for satellite or aerial images. In this article, the proponents present an assessment on the transferability of functionalities for satellite imaging from pre-trained Deep CNNs. Their developed and trained features can be transferred from an unlabelled dataset to a distinct data set. The mix of characteristics taken from a multitude of profound network architectures is explored and evaluated, and more than 2,000 network-layer configurations systematically evaluated. This technique might be helpful when unlabelled pictures and other automated teaching assignments are clustered. The outcome is that these networks can be generalized to other datasets with or without a minimum of practice. Creating an information capacity issue in satellite mapping to identify the contents of pictures is now more spatially, spectrally, and temporally available. Figure 1 shows the sample image patches from the UC Merced Land Use dataset (combined classes). Figure 2 shows the sample image patches from the EuroSAT dataset (combined classes). Because of the performance of $\mathrm{CNN}$ in satellite image classification, the following $\mathrm{CNNs}$ are tested and evaluated for this study.

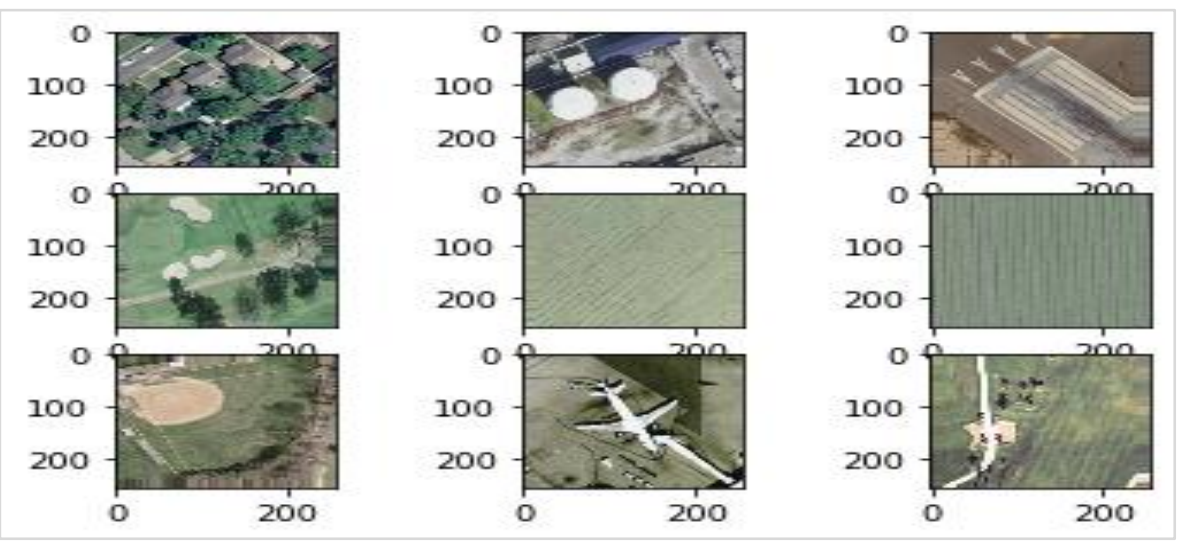

Figure 1 Sample image patches from the UC Merced Land Use dataset (combined classes)

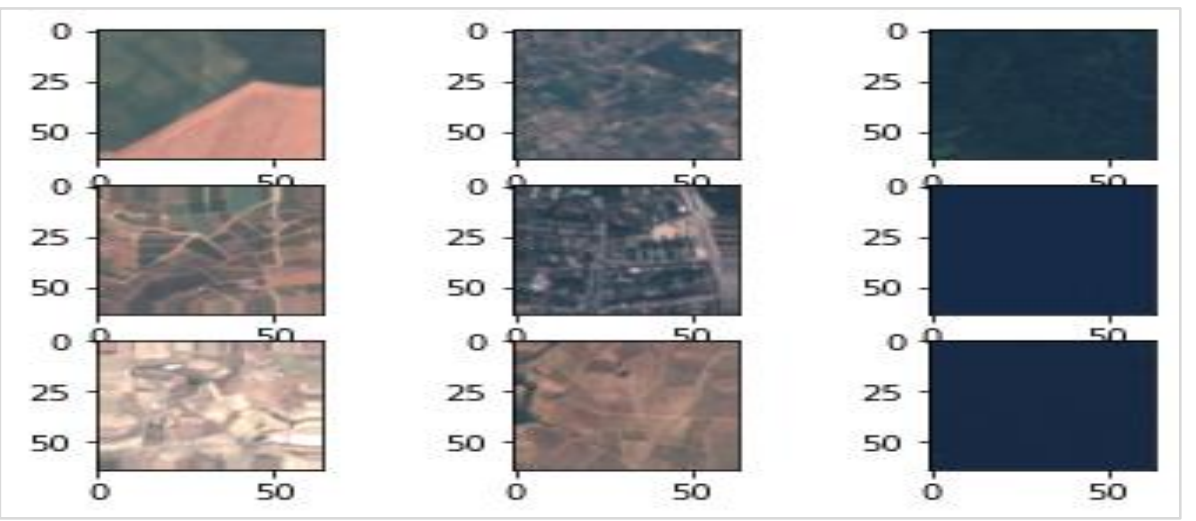

Figure 2 Sample image patches from the EuroSAT dataset (combined classes) 


\section{VGG16}

A convolutional neural network architecture that uses a very small $(3 \times 3)$ convolution filters developed by Velasco et al. [16]. This has a very uniform architecture that consists of 16 convolutional layers [17].

\section{ResNet-50}

A deep residual network with a depth of 50 layers that is developed by Kaiming He, Velasco et al. [16]. This has an architecture with "skip connections", also known as gated recurrent units and has a feature of heavy batch normalization [17].

Development and testing of computational methods are dependent on experimental data. Only in comparison to existing knowledge can method performance be assessed. For that purpose, benchmark datasets with known and verified outcome are needed [18]. Benchmark dataset is used as a standard which solution can be compared and is used both in training and testing.

A study in Germany, Helber et al. [4], proposed a basis for a large-scale training of deep neural networks for the task of satellite image classification. The proponents present EuroSAT, a novel dataset based on Sentinel-2 satellite images covering 13 spectral bands and consisting out of 10 classes with a total of 27,000 labeled and geo-referenced images. They provide benchmarks for this novel dataset with its spectral bands using state-of-the-art deep Convolutional Neural Network (CNNs). With the proposed novel dataset, an overall classification accuracy of $98.57 \%$ is achieved. The resulting classification system opens a gate towards several Earth observation applications. The classification system can be used for detecting land use and land cover changes assist in improving geographical maps.

Another study at the University of California of Yang and Newsam [19], investigates bag-of-visual-words (BOVW) approach to land use classification in highresolution overhead imagery. The proponents evaluated the method using 21 land-use classes selected from aerial orthoimagery with a pixel resolution of one foot. 100 images measuring $256 \times 256$ pixels were manually selected for each of the following 21 classes: agricultural, airplane, baseball diamond, beach, buildings, chaparral, dense residential, forest, freeway, golf course, harbor, intersection, medium density residential, mobile home park, overpass, parking lot, river, runway, sparse residential, storage tanks, and tennis courts.
This is the UC Merced Land Use dataset which is widely used for land use classification. Because of the limited reference data that is openly accessible in the Philippines, the researchers adapted these benchmark datasets for training the CNNs in this study.

In 2018, Fujo and Dida [20], aims to develop a webbased admission system for private schools to replace the existing manual admission system. Likewise, the proponents of this land use classification study aims to develop a web-based land use mapping system that could help in improving the manual procedures in land use classification of LGUs.

Relating land use and web application, Budlong [21] developed a computer administered application that can give automated answers to land related inquiries. Land information, spatial information and city advancement codes, strategy and zoning rules are changed and converted into usable information to produce a usable search. Another example of an application is Disclosed, developed by Harrison [22], which is a land website that offers a custom-made database on which data can be kept, recovered, modified, and transmitted (e.g., by email) linking to a portion of a land. The database can be available via a security key through the Internet and may be scrambled for communication. The said application gives an exclusive means of packaging information involving a portion of property in a sole, available site. Many map data and image data are given to support the user in illustrating the borders.

Web applications in relation to land monitoring have also been developed. In the drought risk mapping study [7], an Internet application has been established for Northern districts of Kerala State in India which can be utilized to strategize for drought supervision measures and can also aid as a record for drought analysis. In 2019, Da et al. [23] developed a mapping system for hydrogeomorphic flood hazard of European rivers which enables users to picture "consistent hydrogeomorphic floodplain delineation at $25 \mathrm{~m}$ spatial resolution and which may evolve into a more powerful tool that attends to users' needs and expectations in supporting evidence-based decision making". These monitoring systems can be compared to weather monitoring web applications, one of Adhy et al. [24] that retrieves data in relatively real-time and present information as the users' needs.

In this study, the land classification system that will be developed can be visualized using a web 
application for, such as in satellite imaging, data visualization is required in displaying the results after extracting information from big data [25]. This web application is aimed to be publicly accessible and everyone that has created an account can access the webpage anytime and anywhere over the Internet in which information can be shown necessary to the user.

\section{Methods}

The aim of this study is to develop a land use classification system utilizing satellite imagery and CNN that is integrated into a web application. Different aspects are considered in acquiring the needed data for this study. The goal is to use open access data that is available. To develop the land use classification system, different satellite imagery tools are considered also as with the benchmark datasets and CNNs that would be used in developing the land use classification web application.

\subsection{Data acquisition}

For acquiring the satellite images, Copernicus Open Access Hub, USGS Earth Explorer and Google Maps are contemplated. Considering that an objective of this study is to create a web-based land mapping system, Google Maps has an Application Programming Interface (API) that can be accessed freely which is used to link the classification system to the web application developed. With this, Google Maps is used in obtaining the satellite images for the land use classification. The table below shows the differences among the open access, satellite imagery portals that the researchers considered.

Because of the limited ground truths/references of areas in the Philippines, two benchmark datasets from the United States and Europe are considered in this study - the UC Merced Land Use dataset and the EuroSAT dataset, respectively. Both can be obtained from the TensorFlow datasets catalogue (https://www.tensorflow.org/datasets/catalog/overvie w).

The benchmark datasets have more categories than the four (4) proposed classifications in this study (agricultural, commercial, industrial, and residential). With this, categories from the datasets which have almost the same features or can be seen in that classification, by visual analysis, are combined which are shown in Tables 2 and 3. The datasets are split into $80 \%$ training and $20 \%$ testing sets class-wise. The table below shows the classes included in the UC Merced Land Use and EuroSAT datasets, and the combined classes for the dataset to be used in the study. Table 1 shows the comparison of open access satellite imagery portals.

Table 1 Comparison of open access satellite imagery portals

\begin{tabular}{|c|c|c|c|}
\hline & Copernicus open access Hub & Google maps & USGS earth explorer \\
\hline Resolution & $10 \mathrm{~m} ; 20 \mathrm{~m} ; 60 \mathrm{~m}$ & $\begin{array}{l}15 \mathrm{~cm} ; 30 \mathrm{~cm} ; 10 \mathrm{~m} ; 15 \mathrm{~m} ; \\
20 \mathrm{~m} ; 30 \mathrm{~m} ; 100 \mathrm{~m}\end{array}$ & 10m; 20m; 30m; 60m \\
\hline Land Area per tile size & $100 \times 100$ sq. km & About 100,000 sq. km & $100 \times 100$ sq. km \\
\hline Download Time & $\begin{array}{l}\text { More than an hour } \\
\text { (API has a subscription) }\end{array}$ & $\begin{array}{l}\text { Linked through API } \\
\text { (API can be accessed freely) }\end{array}$ & $\begin{array}{l}1 \text { hour } \\
\text { (API needs request } \\
\text { access) }\end{array}$ \\
\hline Cloud cover percent & $0 \%$ to $100 \%$ & $<1$ percent & $0 \%$ to $100 \%$ \\
\hline Satellite Used & Sentinel 2 & $\begin{array}{l}\text { Various satellite depending } \\
\text { on the zoom (Landsat, } \\
\text { Sentinel) }\end{array}$ & Landsat, Sentinel 2 \\
\hline Availability & Publicly accessible & Publicly accessible & Publicly accessible \\
\hline
\end{tabular}


Romeo L. Jorda, Jr et al.

Table 2 UC Merced dataset original \& combined class

\begin{tabular}{ll}
\hline UC merced land use dataset & Combined class \\
\hline Original Class & Agricultural \\
\hline Agricultural & \\
Beach & \\
Chaparral & commercial \\
Forest & \\
River & \\
Airplane & \\
Baseballdiamond & \\
Freeway & \\
Golfcourse & \\
Harbor & \\
Intersection & \\
Overpass & \\
Parkinglot & \\
Runway & Residential \\
Tenniscourt & \\
Denseresidential & \\
Mediumresidential & \\
Sparseresidential & \\
Mobilehomes & Industrial \\
Buildings & \\
Storagetanks & \\
\hline
\end{tabular}

Table 3 EuroSAT dataset original \& combined class

\begin{tabular}{ll}
\hline EuroSAT dataset & Combined class \\
\hline Original class & Agricultural \\
\hline Annualcrop & \\
Forest & \\
Herbaceousvegetation & \\
Pasture & \\
Permanentcrop & \\
River & \\
Sealake & Commercial \\
Highway & Industrial \\
Industrial & Residential \\
Residential & \\
\hline
\end{tabular}

\subsection{Convolutional neural network}

The convolutional neural network $(\mathrm{CNN})$ acts as a classifier and recognizer of the system [26-32]. The VGG16 and ResNet-50 are two of the highest performing architectures/top competitors of $\mathrm{CNN}$ that competed in the ImageNet Large Scale Visual Recognition Challenge (ILSVRC) [17]. Thus, these two CNN architectures are considered in this study.

\subsection{Software development}

To be able to maximize the use of the land use classification system that is developed, the system is integrated into a web application. With this, the system would be open for public use, especially for the use of LGUs that could help in the land mapping of their areas. Figure 3 shows the machine learning process starting from the satellite images from Google Maps that is linked to the website. The result of the detection is visualized on the device that is used.

In developing the web application in which the land use classification system would be integrated, also considering the importance of the users in designing the User Experience (UX) [33], the researchers used the following website developing tools in designing the web application:

\section{Front-end user interface}

HTML 5, CSS3 and Bootstrap 3 is used for the positioning of the elements of the webpage and aesthetics. The query is used to make the usage of JavaScript much easier. JavaScript and jQuery are assigned for the events of the page (e.g., what modal will show when a button is clicked), for the display of Google Maps, and for the data to be sent to the server 
for image processing and for the response to be received.

\section{Back-end user interface}

Python and Django are responsible in running the main web server, and in receiving the data from the front-end to process the image (an image that is captured from the map to the image processing) and to return the result to the website. Fast AI is used for image processing.

The process of the land classification system of the developed software is shown in Figure 4. PyhtonAnywhere, which is an online integrated development environment (IDE) that is based on the Python programming language, is used as the web hosting service for the web application to be accessible via Internet. A Google Map display is shown on the web page which is linked through an API. The image is acquired from a screenshot of the map (chosen area of the user) and is sent to the server for image processing. The deep learning library of Fast. AI is used to provide the best results of the image processing. Django, a python-based web framework, is used to run the main server which then receives the data from the front-end to undergo imaging processing, generates a response, and sends it back to the webpage to let the users visualize the result.

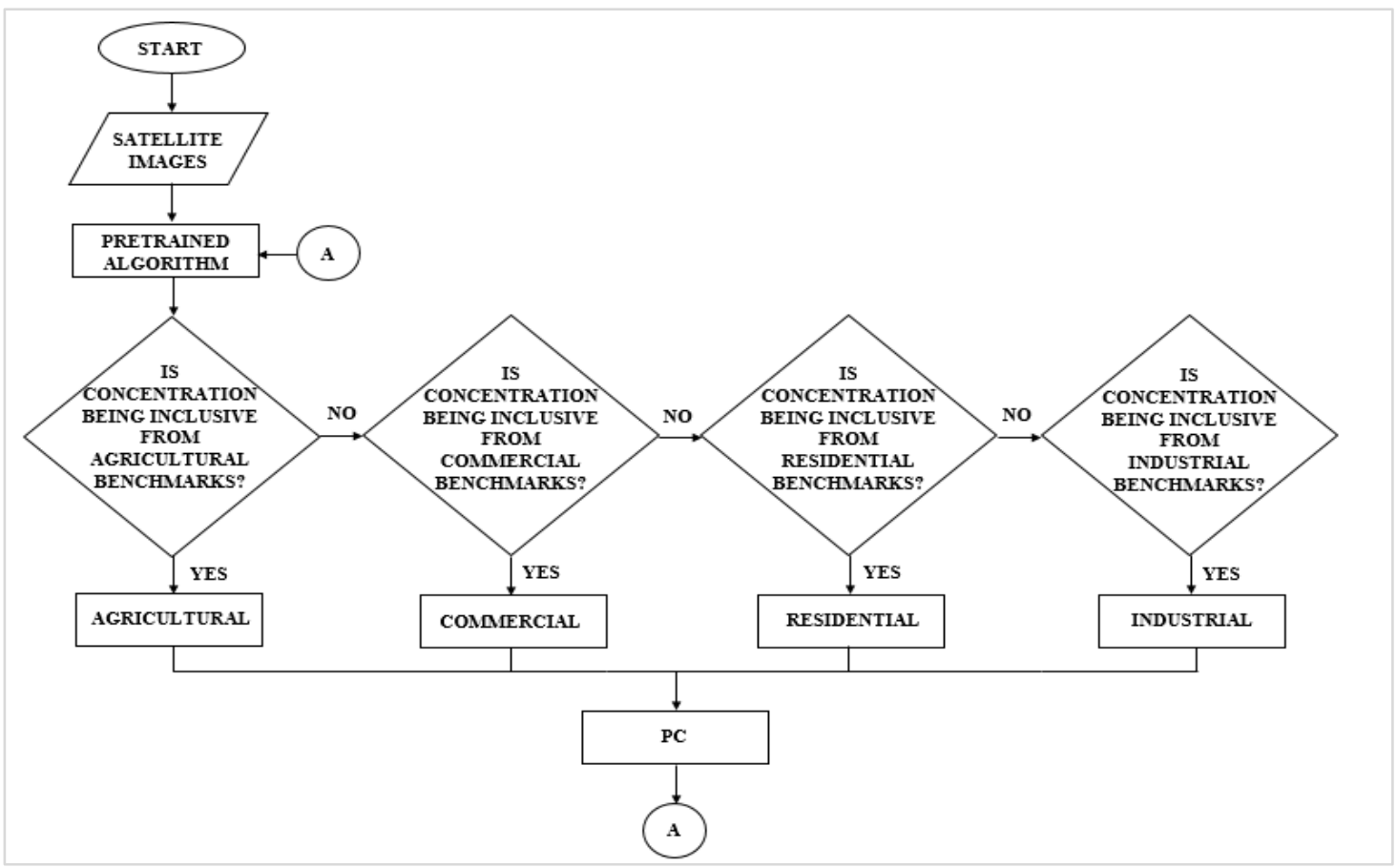

Figure 3 Machine learning process flow chart

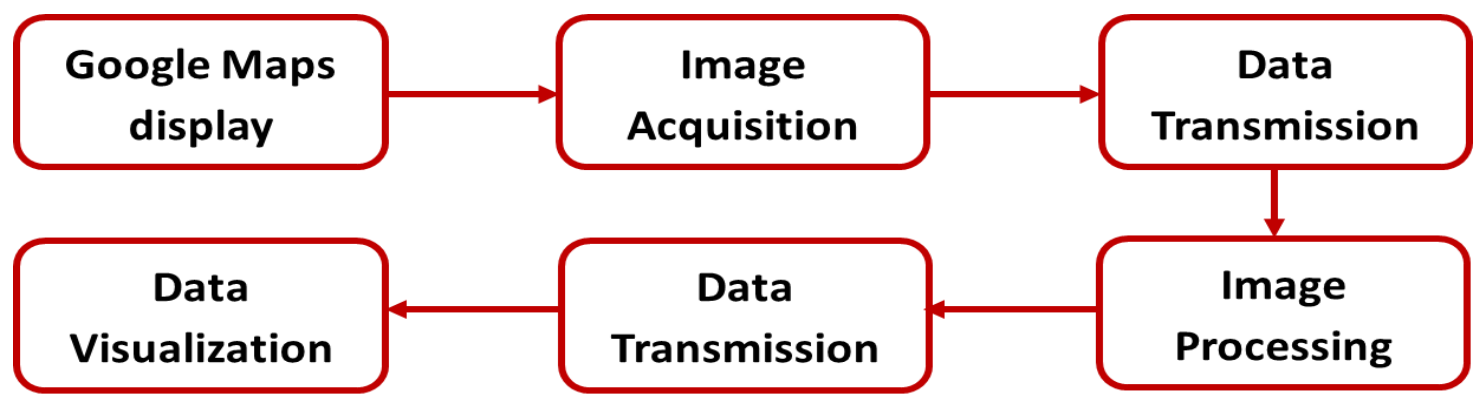

Figure 4 Block diagram of the land classification system of the web application 
Romeo L. Jorda, Jr et al.

\subsection{Testing procedure/fine-tuning of CNN}

The CNNs, VGG16 and ResNet-50, are tested for the satellite image classification. Both are finetuned/transfer learning is applied to both $\mathrm{CNNs}$ on each of the benchmark datasets. Since these are preexisting CNNs, these have already learned the features that are essential to the classification problem that the researchers have in this study. Initial training is done on both CNNs to test their performance and accuracy. The benchmark datasetCNN that will have the better performance will be adapted to the land use classification system. Figure 5 shows the confusion matrix of training ResNet-50 on EuroSAT dataset (combined classes). Figure 6 shows the home page of the developed web application (TUPSI web app). Figure 7 shows the Google map display linked through an API.

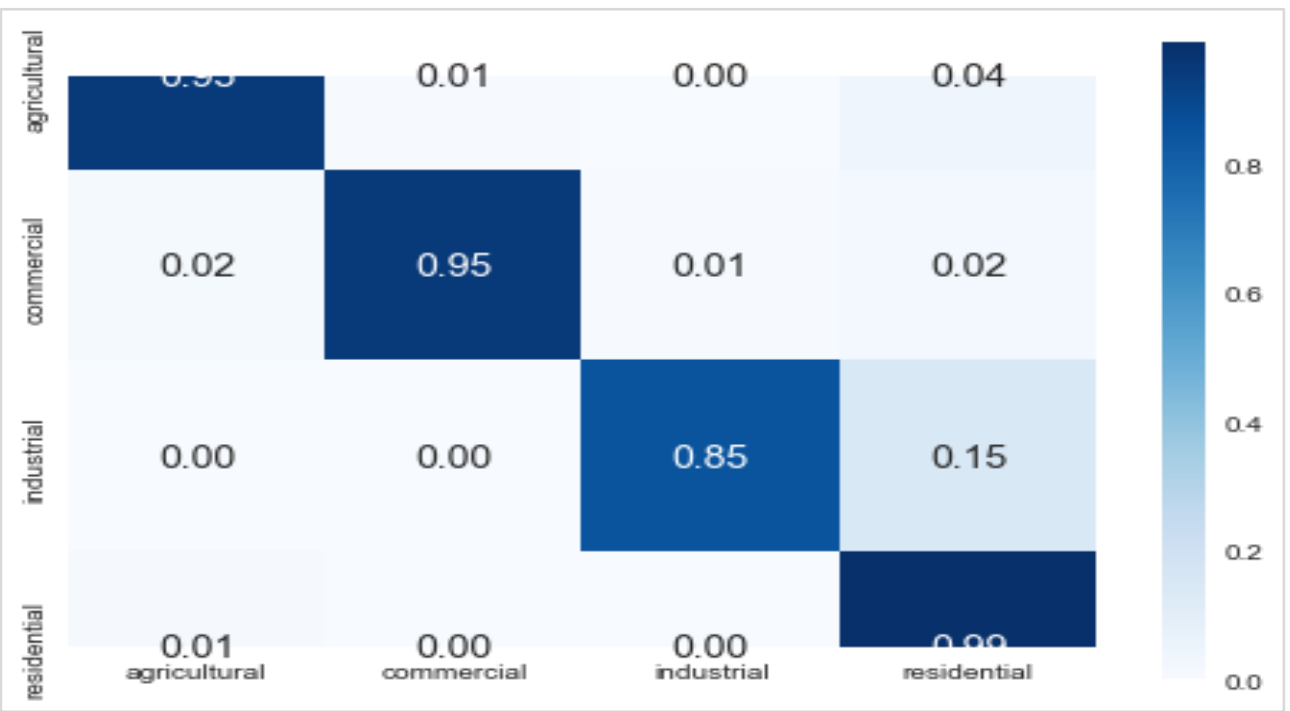

Figure 5 Confusion matrix of training ResNet-50 on EuroSAT dataset (combined classes)

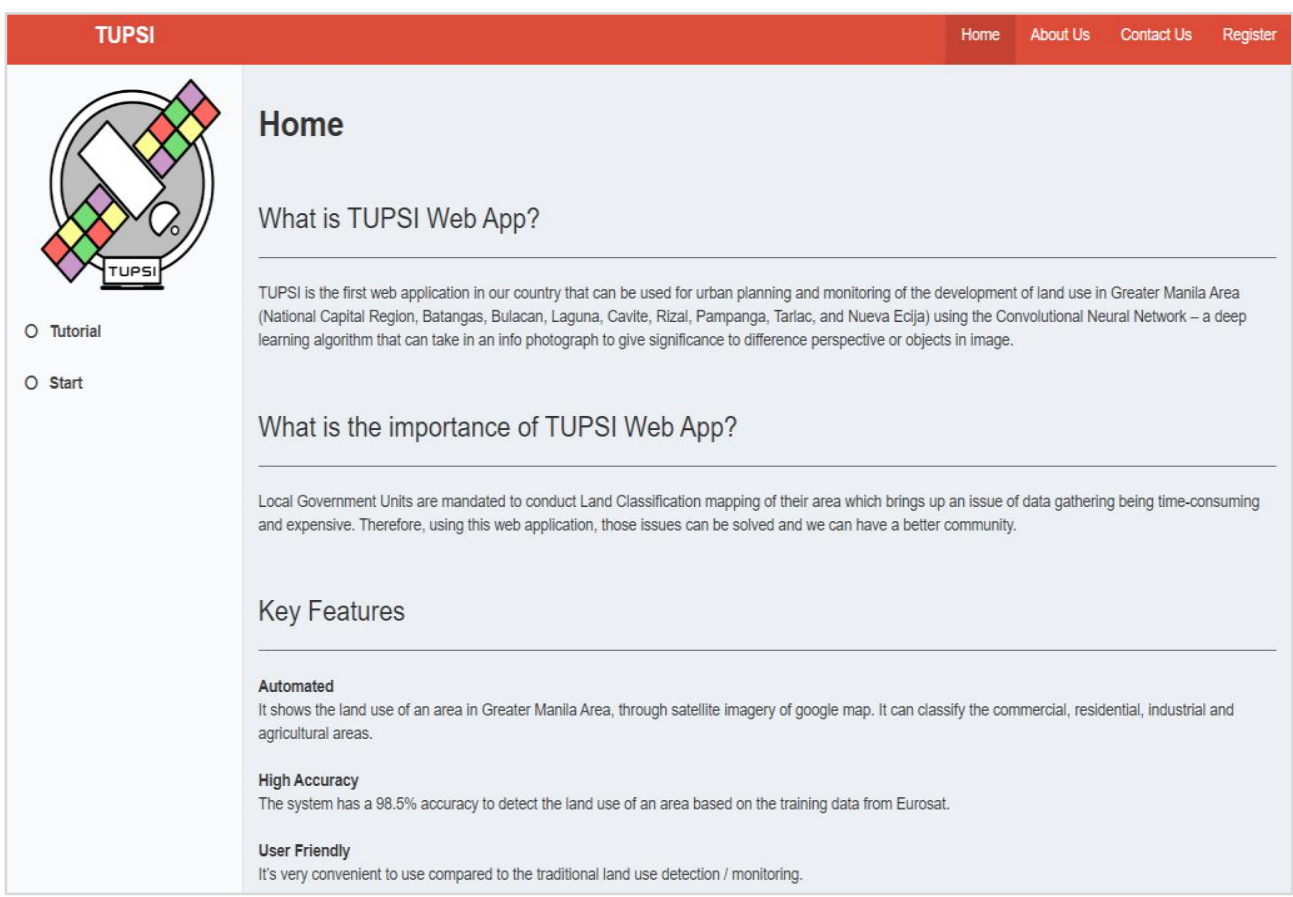

Figure 6 Home page of the developed web application (TUPSI web app) 


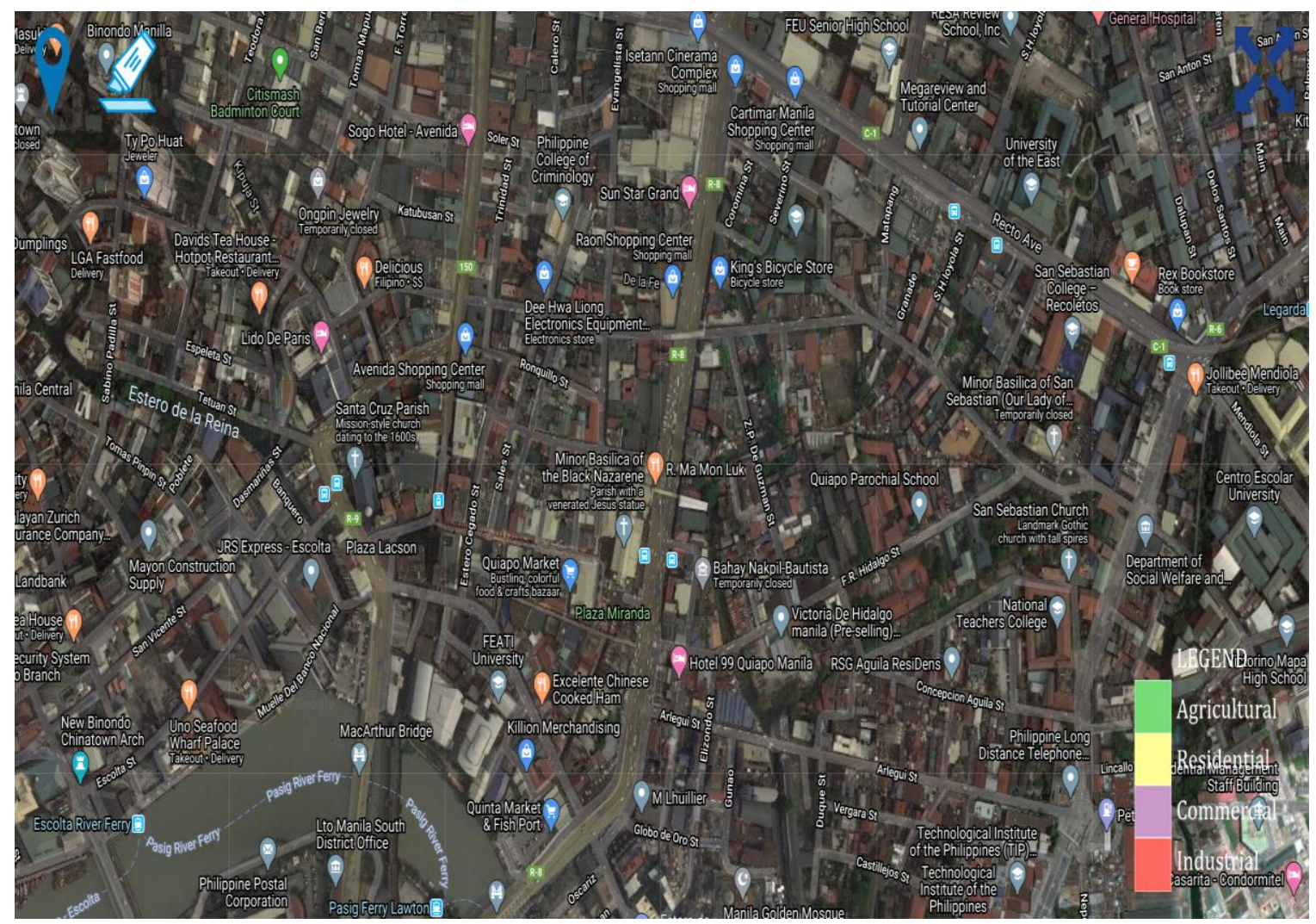

Figure 7 Google map display linked through an API

\section{Results}

\subsection{Testing the CNN}

In the initial training of the CNNs, VGG16 and ResNet-50 are trained each to the UC Merced Land Use dataset (combined classes) and the EuroSAT dataset (combined classes), to assess the performance of the CNNs with different datasets.

After the initial training on each of the datasets, the CNN models are evaluated based on accuracy, precision, recall, and f1-score, which are shown in Tables 4 and 5. ResNet-50 achieved higher performance than VGG16 on both datasets. With these results, ResNet-50 is the CNN model adopted in this study. On the other hand, the EuroSAT dataset (combined classes) is used as the benchmark for the land classification system. Using it as the benchmark together with ResNet-50 has shown significant result of an accuracy of $94.41 \%$ in the initial training than the EuroSAT-VGG16 accuracy of $90.71 \%$

Further fine-tuning of the adapted ResNet-50 on EuroSAT (with an unbalanced data for the combined classes) and tweaking its parameters have resulted in an error rate of $1.53 \%$ for a higher accuracy of $98.47 \%$. This is comparable with the study of Helber, et al., [4] wherein here, the 21-class benchmark EuroSAT dataset is proposed with ResNet-50 being the best performing classifier that achieved the highest accuracy of $98.57 \%$.

\subsection{Evaluation of the web application}

The developed system is evaluated by Information Technology (IT) professionals based on end-user's experience. The following figures and table show the interface of the web application and the evaluation results, respectively.

The interviewed IT experts evaluated the developed web application. The IT experts that answered the online survey (online evaluation form) evaluated the web application based on CNN used and the enduser's experience. Figure 8 shows the location list and the location list drop down menu. Figure 9 shows the sample highlighted map showing the colorcoded classifications. Table 6 shows the further finetuning accuracy of ResNet-50 on the EuroSAT dataset (combined classes) and comparison with the previous study of EuroSAT. Table 7 shows the evaluation results of the web application. 
Romeo L. Jorda, Jr et al.

Table 4 Evaluation of the CNN models on UC Merced dataset (combined classes)

\begin{tabular}{lllll}
\hline CNN & Accuracy & Precision & Recall & F1-Score \\
\hline VGG16 & $47.62 \%$ & 0.12 & 0.25 & 0.16 \\
ResNet-50 & $90.71 \%$ & 0.94 & 0.81 & 0.84 \\
\hline
\end{tabular}

Table 5 Evaluation of the CNN models on EuroSAT dataset (combined classes)

\begin{tabular}{ccccc}
\hline CNN & Accuracy & Precision & Recall & F1-Score \\
\hline VGG16 & $70.37 \%$ & 0.18 & 0.25 & 0.21 \\
ResNet-50 & $94.41 \%$ & 0.91 & 0.93 & 0.91 \\
\hline
\end{tabular}

Table 6 Further fine-tuning accuracy of ResNet-50 on the EuroSAT dataset (combined classes) and comparison with the previous study of EuroSAT

\begin{tabular}{ll}
\hline ResNet-50 & Accuracy \\
\hline Previous study & $98.57 \%$ \\
This study & $98.47 \%$ \\
\hline
\end{tabular}

Table 7 Evaluation results of the web application

\begin{tabular}{lllllll}
\hline S. No & Evaluation questions & Outstanding & $\begin{array}{l}\text { Very } \\
\text { satisfactory }\end{array}$ & Satisfactory & Fair & $\begin{array}{l}\text { Needs } \\
\text { improvement }\end{array}$ \\
\cline { 3 - 7 } & & $\mathbf{5}$ & $\mathbf{4}$ & $\mathbf{3}$ & $\mathbf{2}$ & $\mathbf{1}$ \\
\hline 1 & $\begin{array}{l}\text { How will you rate the CNN used } \\
\text { in the web application? }\end{array}$ & $30.8 \%$ & $61.5 \%$ & $7.7 \%$ & $0 \%$ & $0 \%$ \\
\hline 2 & $\begin{array}{l}\text { How will you rate the loading time } \\
\text { of the web application? }\end{array}$ & $38.5 \%$ & $15.4 \%$ & $38.5 \%$ & $7.7 \%$ & $0 \%$ \\
\hline 3 & $\begin{array}{l}\text { How will you rate the accuracy } \\
\text { of the web application? }\end{array}$ & $23.1 \%$ & $46.2 \%$ & $23.1 \%$ & $7.7 \%$ & $0 \%$ \\
\hline 4 & $\begin{array}{l}\text { What will be your over-all evaluation } \\
\text { of the web application? }\end{array}$ & $15.4 \%$ & $38.5 \%$ & $46.2 \%$ & $0 \%$ & $0 \%$ \\
\hline
\end{tabular}

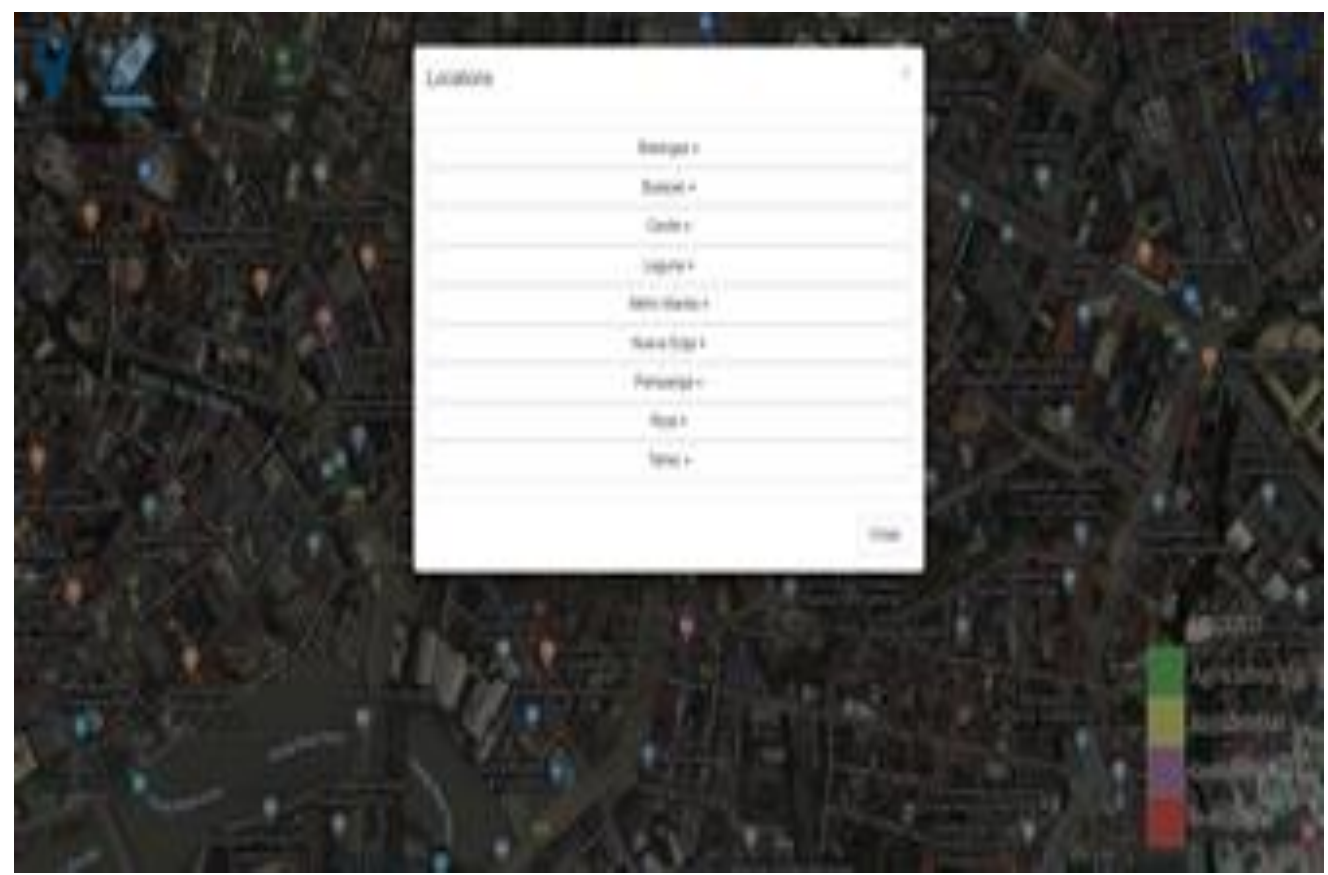

(a) 


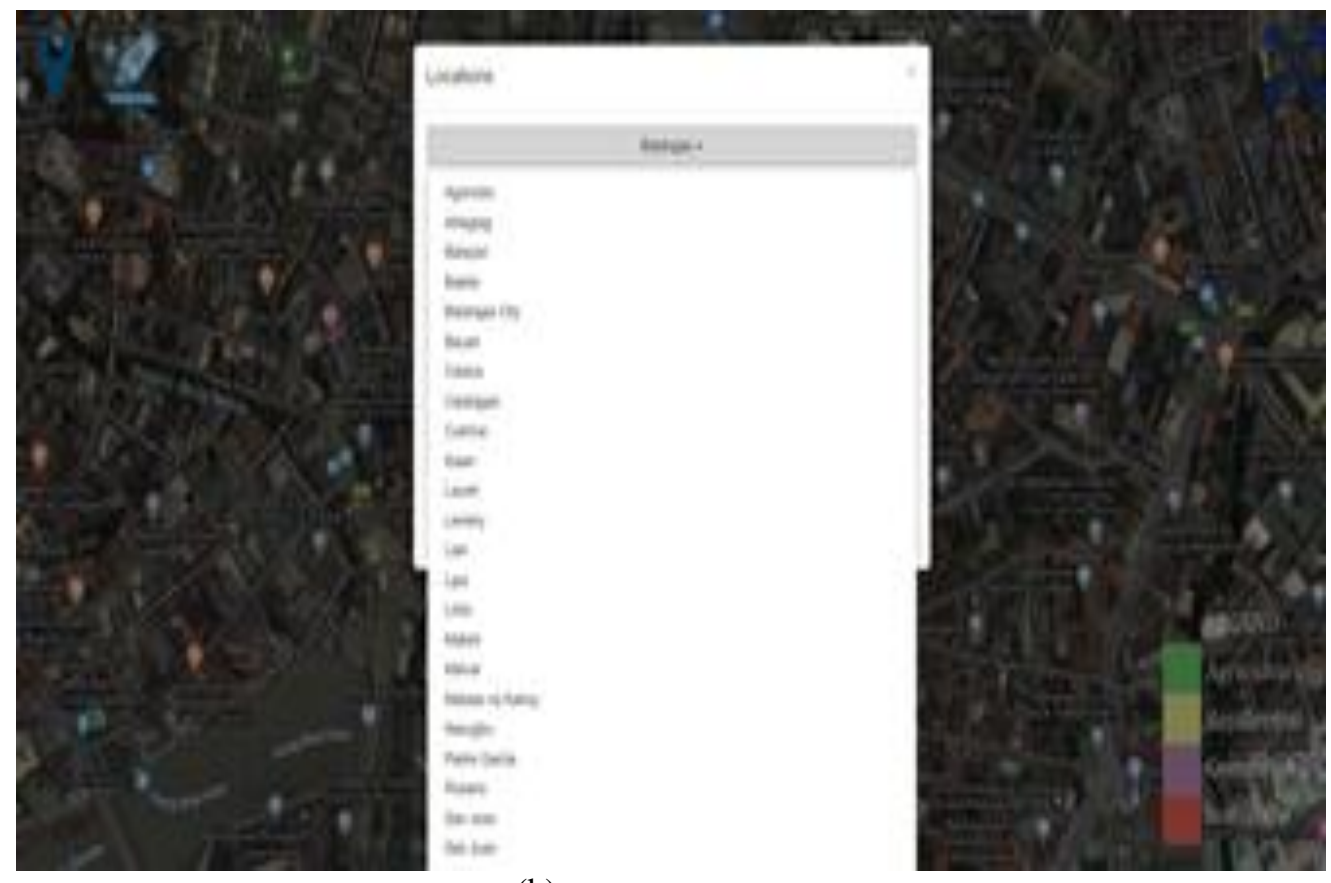

(b)

Figure 8 (a) Location list; (b) location list drop down menu

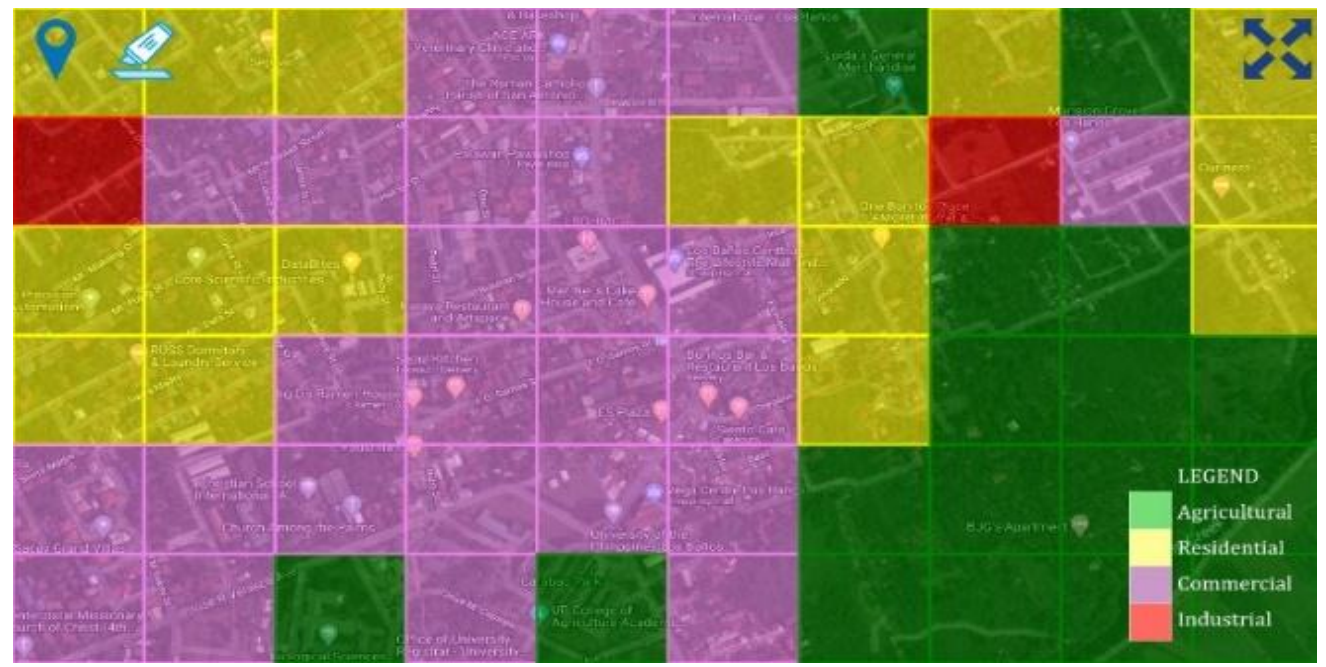

Figure 9 Sample highlighted map showing the color-coded classifications

\section{Discussion}

A land-use mapping web application is developed that utilizes $\mathrm{CNN}$ to train with identified patches to classify the different land-use in different cities and municipalities in the Philippines. Adapted CNN for the study is ResNet-50 which displays higher training error when the depth surges, but can effortlessly gain accurateness from significantly improved depth, creating outcomes which are more improved than the other network.
Based on the data from the evaluation, most users approved the accuracy of the system. On the other hand, a fair view is given with the loading time and over-all evaluation of the web application. As with the user-friendliness of the web application, mostly approves, with some giving suggestions for the improvement of the system. Evaluators approved the usage of the web application for the system could help with the mapping of different areas with the website being simple yet informative and efficient. 


\subsection{Limitations}

The system for the web application has four classification categories: agricultural, residential, commercial, and industrial, and the scope of the study is the Greater Manila Area (Nueva Ecija, Tarlac, Pampanga, Rizal, Metro Manila, Cavite, Bulacan, Laguna and Batangas).

Because of the limited ground truths/references of different areas in the Philippines, this study considered benchmark datasets from the United States and Europe, which resemble scenes of land cover of areas in the Philippines.

As for the web application, an event of classification can be done with the use of the highlight icon which will show the color-coded classification of the land use, and the other one is with the use of the box cursor which will show the classification of the area within the box and the percentage of accuracy. Classification with the use of the box cursor is more accurate than the color-coded classification. Occasionally, some functions fail when using internet browsers other than Google Chrome because of the languages used for web creation. Also, as the web application relies on the use of the internet, the loading time of the classification may be affected by slow internet connection. Using the web application in mobile phones can affect the classification because of the smaller screen size.

\section{Conclusion and future work}

In this paper, a more cost-efficient and less timeconsuming way of land use mapping is presented through a land use classification system that is integrated in a web application. Furthermore, a solution to the limited availability of data of land use classification in the areas of the Philippines is presented in which various aspects of the acquisition of data required in the study is considered and tested. This is to be able to choose the best classification to be used in the integration of the system with a web application.

Integrating the land use classification system on a web application and making it for public use can help, especially government institutions with their urban planning or resource allocations.

While many studies have already manifested great results with the use of satellite imagery and machine learning in land use classification, it is a significant way of showing that utilizing freely available satellite data and machine learning in different applications can help in providing useful data for the improvement of the country.

\subsection{Future work}

For further betterment of this study and for future works, the following are recommended:

- The land classification system can be improved with the availability of ground truths covering Philippine scenes. Further studies could help identify other datasets for better classification of the different land use in the Philippines with collection of additional data and update in mapping details.

- The User Interface (UI) of the web application can be enhanced more for a better user experience (best viewing and navigation) and the availability of the UI of the system for mobile application to be accessible to everyone.

- This study can also be improved for distinguishing, surveying, and reducing the risks of disaster given that roads and homes in most of the cities in the Greater Manila area immediately flood when it rains continuously. A real-time map is preferable to foresee the changes in land cover immediately.

\section{Acknowledgment}

The authors would like to thank the Technological University of the Philippines for the support and funding.

\section{Conflicts of interest}

The authors have no conflicts of interest to declare.

\section{References}

[1] https://www.officialgazette.gov.ph/1992/03/24/republi c-act-no-7279/. Accessed 01 August 2020.

[2] Castelluccio M, Poggi G, Sansone C, Verdoliva L. Land use classification in remote sensing images by convolutional neural networks. arXiv preprint arXiv:1508.00092. 2015:1-11.

[3] Bischke B, Borth D, Schulze C, Dengel A. Contextual enrichment of remote-sensed events with social media streams. In proceedings of the ACM international conference on Multimedia 2016 (pp. 1077-81).

[4] Helber P, Bischke B, Dengel A, Borth D. Eurosat: A novel dataset and deep learning benchmark for land use and land cover classification. IEEE Journal of Selected Topics in Applied Earth Observations and Remote Sensing. 2019; 12(7):2217-26.

[5] Omollo R, Alago S. Data modeling techniques used for big data in enterprise networks. International Journal of Advanced Technology and Engineering Exploration. 2020; 7(65):79-92.

[6] Inggs MR, Lord RT. Applications of satellite imaging radar. South African Institute of Electrical Engineers, SAIEE, South Africa. 2000:1-8. 
[7] Gopinath G, Ambili GK, Gregory SJ, Anusha CK. Drought risk mapping of south-western state in the Indian peninsula-A web based application. Journal of Environmental Management. 2015; 161:453-9.

[8] https://phl-microsat.upd.edu.ph/. Accessed 08 August 2020.

[9] Anderson C, Labahn S, Helder D, Stensaas G, Engebretson C, Crawford C, et al. The US geological survey's approach to analysis ready data. In international geoscience and remote sensing symposium 2019 (pp. 5541-4). IEEE.

[10] Vandeviver C. Applying google maps and google street view in criminological research. Crime Science. 2014; 3(1):1-6.

[11] LeCun Y, Bengio Y, Hinton G. Deep learning. Nature. 2015; 521(7553):436-44.

[12] Luo W, Li Y, Urtasun R, Zemel R. Understanding the effective receptive field in deep convolutional neural networks. In proceedings of the international conference on neural information processing systems 2016 (pp. 4905-13). ACM.

[13] Tanuja K, Usha Kumari V, Sushma TM. Handwritten Hindi character recognition system using edge detection \& neural network. International Journal of Advanced Technology and Engineering Exploration.2015; 2(6):71-5.

[14] Jean N, Burke M, Xie M, Davis WM, Lobell DB, Ermon S. Combining satellite imagery and machine learning to predict poverty. Science. 2016; 353(6301):790-4.

[15] Hedayatnia B, Yazdani M, Nguyen M, Block J, Altintas I. Determining feature extractors for unsupervised learning on satellite images. In international conference on big data (Big Data) 2016 (pp. 2655-63). IEEE.

[16] Velasco JS, Cabatuan MK, Dadios EP. Urine sediment classification using deep learning. Lecture Notes on Advanced Research in Electrical and Electronic Engineering Technology. 2019:180-5.

[17] https://medium.com/analytics-vidhya/cnnsarchitectures-lenet-alexnet-vgg-googlenet-resnet-andmore-666091488df5. Accessed 08 August 2020.

[18] Sarkar A, Yang Y, Vihinen M. Variation benchmark datasets: update, criteria, quality and applications. Database. 2020:1-16.

[19] Yang Y, Newsam S. Bag-of-visual-words and spatial extensions for land-use classification. In proceedings of the SIGSPATIAL international conference on advances in geographic information systems 2010 (pp. 270-9).

[20] Fujo MH, Dida MA. Web-based admission system for advanced level, private schools: case of Kilimanjaro region, Tanzania. International Journal of Advanced Technology and Engineering Exploration.2018; 5(47):407-18.

[21] Budlong L, inventor. Methods for the transformation of complex zoning codes and regulations to produce usable search. United States Patent US 10,074,145. 2018.
[22] Harrison C, inventor; LandNet Corp, assignee. Identification, storage and display of land data on a website. United States Patent US 7,171,389. 2007.

[23] Da Costa RT, Manfreda S, Luzzi V, Samela C, Mazzoli P, Castellarin A, et al. A web application for hydrogeomorphic flood hazard mapping. Environmental Modelling \& Software. 2019; 118:17286.

[24] Adhy S, Noranita B, Kusumaningrum R, Wirawan PW, Prasetya DD, et al. Usability testing of weather monitoring on a web application. In international conference on informatics and computational sciences 2017 (pp. 131-6). IEEE.

[25] Joshi P. Impact of big data on computer graphics. International Journal of Advanced Technology and Engineering Exploration. 2017; 4(32):117-22.

[26] Tolentino LK, Juan RS, Thio-ac AC, Pamahoy MA, Forteza JR, Garcia XJ. Static Sign language recognition using deep learning. International Journal of Machine Learning and Computing. 2019; 9(6):8217.

[27] Arago NM, Alvarez CI, Mabale AG, Legista CG, Repiso NE, Robles RR, et al. Automated estrus detection for dairy cattle through neural networks and bounding box corner analysis. International Journal of Advanced Computer Science and Applications. 2020; 11(9):303-11.

[28] Bague LR, Romeo Jr L, Fortaleza BN, Evanculla AD, Paez MA, Velasco JS. Recognition of baybayin (Ancient Philippine Character) handwritten letters using VGG16 deep convolutional neural network model. International Journal of Emerging Trends in Engineering Research. 2020; 8(9):5233-7.

[29] Velasco J, Ang JR, Caibigan J, Naval FM, Arago N, Fortaleza B. Identification of normal and diseased lungs using X-ray images through transfer learning. International Journal of Advanced Trends in Computer Science and Engineering. 2020; 9(4): 6227-31.

[30] Velasco JS, Arago NM, Mamba RM, Padilla MV, Ramos JP, Virrey GC. Cattle sperm classification using transfer learning models. International Journal of Emerging Trends in Engineering Research. 2020; 8(8): 4325-31.

[31] Velasco JS, Padilla MV, Arago NM, De Vera EP, Domingo FE, Ramos RE. Canine semen evaluation using transfer learning models. International Journal of Emerging Trends in Engineering Research. 2020; 8(7): 4013-8.

[32] Velasco J, Pascion C, Alberio JW, Apuang J, Cruz JS, Gomez MA, et al. A smartphone-based skin disease classification using mobilenet $\mathrm{CNN}$. International Journal of Advanced Trends in Computer Science and Engineering. 2019; 8(5):2632-7.

[33] Mambile C, Machuve D, Moebs S. User experience of a web-based platform for poultry market linkages in Tanzania. International Journal of Advanced Technology and Engineering Exploration. 2018; 5(49):495-503. 


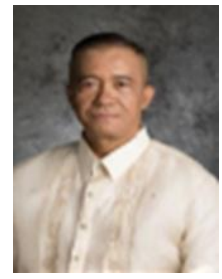

Romeo L. Jorda, Jr. was the Head of the Department of Electronics Engineering, Technological University of the Philippines (TUP) in Manila from 2001 to 2012. He was the College Secretary at the College of Engineering, TUP, from 2012 to 2015. He is an Assistant Professor at TUP. He is currently one of the members of the Technical Committee on Audio, Video, and Multimedia Equipment (TC 59) of the Bureau of Product Standards of the Philippines' Department of Trade and Industry. $\mathrm{He}$ is a licensed Professional Electronics Engineer and a certified ASEAN Engineer. He is an active member of IEEE and IECEP. Email: Romeo_jorda@tup.edu.ph

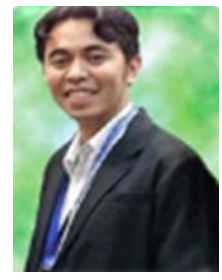

Edmon O. Fernandez is currently the Head of the Department of Electronics Engineering, Technological University of the Philippines (TUP) in Manila since 2019. He is concurrently an Associate Professor at TUP and is also one of the members of the Technical Committee on Electromagnetic Compatibility (TC 74) of the Bureau of Product Standards of the Philippines' Department of Trade and Industry. He graduated his Master of Science in Electrical Engineering Program in TUP - Manila. Presently, he is taking up his $\mathrm{PhD}$ in Electronics Engineering at the Mapua University. His research interest is in signal processing and control system.

Email: edmon_fernandez@tup.edu.ph

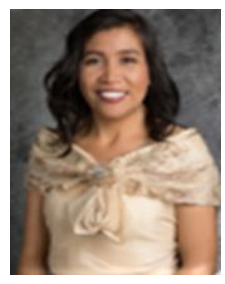

Jessica S. Velasco is an Assistant Professor in the Department of Electronics Engineering at the Technological University of the Philippines. She was the Head of the Department of Electronics Engineering, TUP, from 2016 to 2017. She has 15 years of teaching/research. She graduated her Master's degree in Electronics Engineering at Gokongwei College of Engineering from De La Salle University (DLSU), Manila. Presently, she is taking up her $\mathrm{PhD}$ in Electronics Engineering in DLSU, also. Her areas of interest include artificial intelligence and machine learning.

Email: jessica_velasco@tup.edu.ph

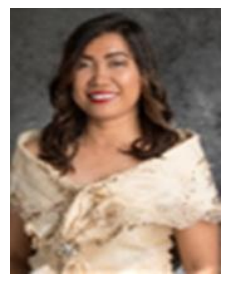

Maria Victoria C. Padilla is currently a Faculty Member of the Department of Electronics Engineering at Technological University of the Philippines. She graduated MS in Electronics Engineering major in Microelectronics at the Mapua University. She is currently one of the members of the Technical Committee on Cleanrooms (TC 83) of the Bureau of Product Standards of the Philippines'
Department of Trade and Industry. Her areas of interest include biomedical engineering and sensor systems.

Email: mariavictoria_padilla@tup.edu.ph

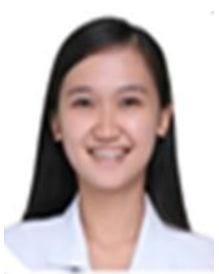

Shiela Mae P. Agoylo finished her studies in high school at the Zambales National High School under the Special Science Program. She was a Department of Science and Technology scholar and finished her bachelor's degree in Electronics Engineering major in Communications at the Technological University of the Philippines - Manila and graduated last 2020.

Email: shielamae.agoylo@tup.edu.ph

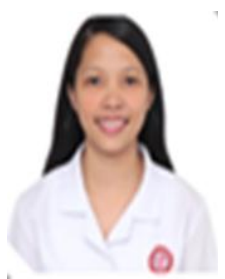

Mary Joy A. Bultron took first a 3year vocational course, Electronics Engineering Technology (ESET) at the Technological University of the Philippines - Manila last 2016. She decided to pursue it to a Bachelor of Science in Electronics Engineering (BSECE) at the same university and graduated August 2020. She is a Licensed Electronics Technician, and she is currently a Project Coordinator in Asiatrend Marketing Inc., a telecommunication company under of Huawei.

Email: maryjoy.bultron@tup.edu.ph

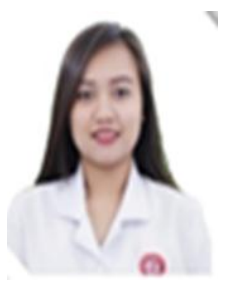

Ailyn Joyce O. Clamor finished Electronics Engineering Technology, a 3-year vocational course at Technological University of the Philippines - Cavite last 2016. She was able to pursue a bachelor's degree in Electronics Engineering major in Microelectronics at the Technological University of the Philippines - Manila. She became a licensed Electronics Technician before graduating last 2020.

Email: ailynjoyce.clamor@tup.edu.ph

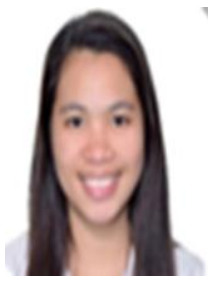

Jaishree Keith M. Monzaga finished her studies in high school at the Holy Nazarene Christian School. She became a varsity player and the captain ball of the women's basketball team of the Technological University of the Philippines. She was able to pursue a bachelor's degree in Electronics Engineering major in Communications at Technological University of the Philippines - Manila and graduated last 2020

Email: jaishreekeith.monzaga@tup.edu.ph 


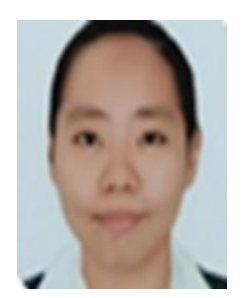

Catherine Rose R. Rosales finished secondary education at the Parañaque Science High School and is a graduate of BS Electronics Engineering at the Technological University of the Philippines, Manila. She was also a Department of Science and Technology Undergraduate Scholarship Awardee. In 2018, she passed the Electronics Technician Licensure Exam.

Email: catherinerose.rosales@tup.edu.ph

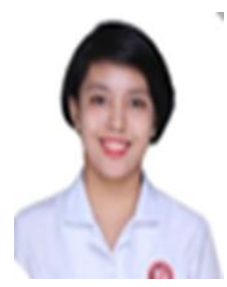

Cleodelaine S. Salvador finished secondary school at the Caybiga High School. She finished Bachelor of Science in Electronics Engineering major in Communications at the Technological University of the Philippines - Manila and graduated last 2020.

Email: cleodelaine.salvador@tup.edu.ph

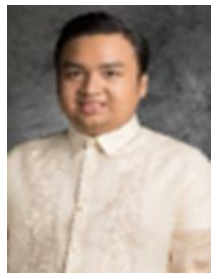

Lean Karlo S. Tolentino was the Head of the Department of Electronics Engineering, Technological University of the Philippines (TUP) in Manila from 2017 to 2019 . He is currently the director of the University Extension Services Office and Assistant Professor at TUP since 2019 . He is currently one of the members of the Technical Committee on Audio, Video, and Multimedia Equipment (TC 59) and Technical Committee on Electromagnetic Compatibility (TC 74) of the Bureau of Product Standards of the Philippines' Department of Trade and Industry. He is a Regular Member of the National Research Council of the Philippines. He is an active member of IEEE and IECEP. His research interests include microelectronics and information and computing technologies.

Email: leankarlo_tolentino@tup.edu.ph 